\title{
Weak emission line central stars of planetary nebulae $\dagger$
}

\author{
Helga Todt ${ }^{1}$, Miriam Peña ${ }^{2}$, Julia Zühlke ${ }^{1}$, Lida Oskinova ${ }^{1}$, \\ Wolf-Rainer Hamann ${ }^{1}$ and Götz Gräfener ${ }^{3,1}$ \\ ${ }^{1}$ Institut für Physik und Astronomie, Universität Potsdam, 14476 Potsdam, Germany \\ email: htodt@astro.physik.uni-potsdam.de \\ ${ }^{2}$ Instituto de Astronomía, Universidad Nacional Autónoma de México, Apdo. Postal 70264, \\ México D.F. 04510, México \\ ${ }^{3}$ Armagh Observatory, College Hill, Armagh BT61 9DG, Northern Ireland
}

\begin{abstract}
To understand the evolution and morphology of planetary nebulae, a detailed knowledge of their central stars is required. Central stars that exhibit emission lines in their spectra, indicating stellar mass-loss allow to study the evolution of planetary nebulae in action. Emission line central stars constitute about $10 \%$ of all central stars. Half of them are practically hydrogen-free Wolf-Rayet type central stars of the carbon sequence, [WC], that show strong emission lines of carbon and oxygen in their spectra. In this contribution we address the weak emission-lines central stars (wels). These stars are poorly analyzed and their hydrogen content is mostly unknown. We obtained optical spectra, that include the important Balmer lines of hydrogen, for four weak emission line central stars. We present the results of our analysis, provide spectral classification and discuss possible explanations for their formation and evolution.
\end{abstract}

Keywords. stars: AGB and post-AGB, stars: Wolf-Rayet, stars: abundances

The class of weak emission-lines stars (wels) was introduced by Tylenda et al. (1993) to tell these emission-lines central stars from the [WC] central stars, which show much stronger and broader emission lines in their optical spectra. Within a sample of 77 emission line CS Tylenda et al. (1993) classified 38 CSPNe as wels. In contrast to the [WC] stars the wels are not systematically analyzed so far. We obtained mid- and highresolution optical spectra of seven wels and perform spectral analysis using PoWR NLTEstellar atmosphere models (Gräfener et al. 2002; Hamann \& Gräfener 2003; Hamann \& Gräfener 2004) to derive stellar mass-loss rate, effective temperature, and chemical composition.

PB 8 was initially classified as a wels, and also as [WC5-6] by Acker \& Neiner (2003). We analyze a high-resolution optical spectrum of PB 8, which was obtained with the Clay $6.5 \mathrm{~m}$-telescope and the double échelle spectrograph MIKE (Magellan Inamori Kyocera Echelle). We find that the CS is H-deficient with a strong wind, but it has not a [WC] like composition (Todt et al. 2010). Instead the composition resembles those of massive WN/WC stars with H:He:C:N:O=40:55:1:2:1 (mass fraction). Therefore we introduce a new class of [WN/WC]-type central stars with PB 8 as its first member. Interestingly, the surrounding PN seems to be young, i.e. $t_{\mathrm{dyn}}<3000 \mathrm{a}$, and is not a Peimbert's Type I PN (García-Rojas et al. 2009). Miller Bertolami et al. (2011) suggested that PB 8 was possibly formed by the occurrence of a diffusion-introduced nova.

Our optical spectra of the other wels have a much lower spectral resolution and a lower $\mathrm{S} / \mathrm{N}$ ratio. The results we report here are only preliminary. In the case of NGC 6572 we

$\dagger$ This paper includes data gathered with the 6.5-m Magellan Telescopes located at Las Campanas Observatory, Chile. 


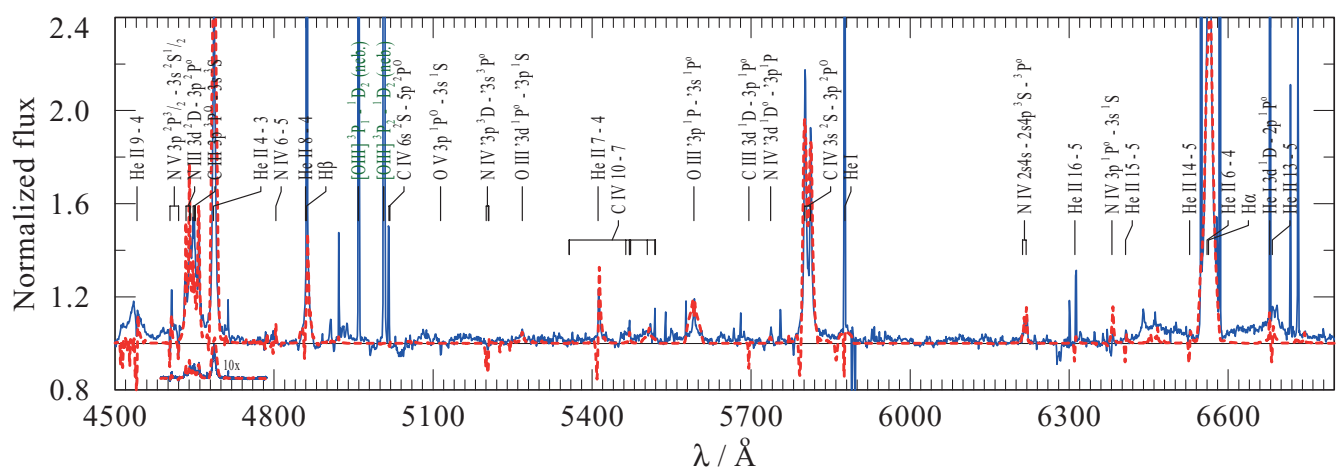

Figure 1. Optical spectrum of PB 8 - Observation (blue solid) vs. model (red dashed)

obtain stellar and wind parameters similar to PB 8. NGC 6572 is therefore a [WN/WC] candidate. For NGC 6891 we infer a hydrogen abundance of about $20 \%$ from the strengths of the absorption lines of the Pickering and Balmer series. The carbon abundance is about 1\%. Abell 30 (alias A66 30) and Abell 78 (alias A66 78) have $X_{\mathrm{C}}=20 \%$ and are practically $\mathrm{H}$-free. Both stars are also classified as [WC]-PG 1159 transit type CS (Werner 1992). SwSt 1 is a cool [WC9] CS with $X_{\mathrm{C}}=50 \%$, as found by Leuenhagen \& Hamann (1998). The emission lines appear weak and narrow, the latter is because of the low wind velocity of only $v_{\infty}=400 \mathrm{~km} \mathrm{~s}^{-1}$. NGC 6543 was first analyzed by Georgiev et al. (2008). They found that although there are emission lines of carbon in the optical spectrum, the stellar wind has a chemical composition which is very similar to solar. This is in agreement with the results of our analysis.

Quantitative spectral analyses by means of sophisticated NLTE code reveal that wels are not a uniform class of CSPNe. The wels classification is ambiguous and a classification based on spectral analysis is preferred. Based on our analysis of PB 8 a new class [WN/WC] is defined and NGC 6572 is a new candidate of this class. It is still an open question, why some CSPNe show strong emission lines in their spectra while others do not. For the massive stars it is the proximity to the Eddington limit, i.e. the $L / M$ ratio, that determines the mass-loss rate and hence the occurrence of emission lines in the optical spectra. Unfortunately reliable $L$ and $M$ measurements for most of the CSPNe are not available.

\section{Acknowledgements}

H. Todt was supported by the DAAD (German Academic Exchange Service).

\section{References}

Acker, A. \& Neiner, C. 2003, A\&A 403, 659

García-Rojas, J., Peña, M., \& Peimbert, A. 2009, A\&̈A 496, 139

Georgiev, L. N., Peimbert, M., Hillier, D. J., Richer, M. G., Arrieta, A., \& Peimbert, A. 2008, ApJ 681, 333

Gräfener G., Koesterke L., \& Hamann W.-R. 2002, A\& $A$ 387, 244

Hamann, W.-R. \& Gräfener, G. 2003, A\&A 410, 993

Hamann, W.-R. \& Gräfener, G. 2004, $A \& A$ 427, 697

Leuenhagen, U. \& Hamann, W.-R. 1998, A\&A 330, 265

Miller Bertolami, M. M., Althaus, L. G., Olano, C., \& Jiménez, N. 2011, MNRAS 415, 1396

Todt, H., Peña, M., Hamann, W.-R., \& Gräfener, G. 2010 A $\mathscr{E} A$, 515, 83

Tylenda, R., Acker, A., \& Stenholm, B. 1993, AAPS 102, 595

Werner, K. 1992 in: U. Heber \& C. S. Jeffery (eds.), The Atmospheres of Early-Type Stars Lecture Notes in Physics 401 (Berlin: Springer Verlag), p. 273 